\title{
Correspondence
}

\section{Constructive Interference as an Information Carrier by Dual-Layered MIMO Transmission}

3 Christos Masouros, Senior Member, IEEE, and

4 Lajos Hanzo, Fellow, IEEE

5 Abstract-We propose a bandwidth-efficient transmission scheme for 6 multiple-input-multiple-output point-to-point and downlink channels. 7 The bandwidth efficiency (BE) of spatial multiplexing (SMX) is improved 8 by implicitly encoding information in the spatial domain based on the exis9 tence of constructive interference in the received symbols, which creates a 10 differentiation in the symbol power. Explicitly, the combination of symbols 11 received at a higher power level carries implicit information in the spatial 12 domain in the same manner as that the combination of nonzero elements in 13 the received symbol vector carries information for receive-antenna-based 14 spatial modulation (RSM). The nonzero power throughout the received 15 symbol vector for the proposed technique allows a full SMX underlying 16 transmission, with the BE enhancement brought by the spatial symbol. 17 Our simulation results demonstrate both significant BE gains and error 18 probability reduction for our approach over the conventional SMX and 19 RSM schemes.

20 Index Terms-Multiple-input multiple-output (MIMO), precoding, spa21 tial modulation (RSM), spatial multiplexing (SMX).

\section{INTRODUCTION}

23 Multiple-input multiple-output (MIMO) systems have been shown 24 to improve the capacity of the wireless channel by means of spatial 25 multiplexing (SMX). Transmit precoding (TPC) schemes introduced 26 for multiuser downlink (DL) transmission improve both the power 27 efficiency and cost of mobile stations by shifting the signal processing 28 complexity to the base stations. From the wide range of linear and 29 nonlinear TPC schemes found in the literature, here, we focus our 30 attention on the family of closed-form linear TPC schemes based on 31 channel inversion [1], [2], which pose low computational complexity. 32 More recently, spatial modulation (SM) has been explored as a means 33 of implicitly encoding information in the index of the specific transmit 34 antenna (TA) activated for the transmission of the modulated symbols, 35 which offers a low-complexity design alternative [3]. Its central bene36 fits include the absence of interantenna interference and the fact that, 37 in contrast to SMX, it only requires a subset (down to one) of radio38 frequency chains compared with SMX. Early work has focused on the 39 design of receiver algorithms for minimizing the bit error ratio (BER) 40 of SM at low complexity [3]-[5].

41 In addition to receive processing, recent work has also proposed 42 constellation shaping for SM [6]-[14]. Specifically, the contributions 43 on this topic have focused on three main directions: 1) shaping and

Manuscript received June 20, 2015; revised November 3, 2015; accepted February 8, 2016. This work was supported in part by the U.K. Royal Academy of Engineering and in part by the Engineering and Physical Sciences Research Council through Project EP/M014150/1. The review of this paper was coordinated by Prof. W. A. Krzymień.

C. Masouros is with London WC1E 7JE, U.K. (e-mail: c.masouros@ucl.ac. uk).

L. Hanzo is with the School of Electronics and Computer Science, University of Southampton, Southampton SO17 1BJ, U.K. (e-mail: 1h@ecs.soton.ac.uk).

Color versions of one or more of the figures in this paper are available online at http://ieeexplore.ieee.org.

Digital Object Identifier 10.1109/TVT.2016.2528506 optimization of the spatial constellation, i.e., the legitimate sets of 44 activated TAs [6]; 2) modulation constellation shaping [7]-[9] for the 45 SM transmission where the constellation of the classically modulated 46 bits is optimized; and 3) joint spatial and modulation constellation 47 shaping, in the form of optimizing the received constellation [10]-[14]. 48 Closely related treatises have been focused on applying SM to the 49 receive antennas (RAs) of the communication link, forming the RA- 50 based spatial modulation (RSM) regime [15], [16]. By means of 51 precoding at the transmitter, this regime aims at transmitting to a 52 reduced a subset of RAs that receive information symbols, whereas the 53 rest of the antennas receive only noise. A dual-layered transmission 54 (DLT) scheme was proposed in [17], where the spatial symbol is 55 conveyed, not by transmitting a combination of symbols and zeros 56 but by assigning a pair of power levels $\left\{P_{1}, P_{2}\right\}$ to the received 57 symbols, with the combination of power levels detected at the receiver 58 representing a spatial symbol.

Here, we explore a power-efficient alternative, where the distinction 60 of the power levels in DLT is no longer formed by the aforementioned 61 direct power allocation but rather by allowing the constructive interfer- 62 ence to form a subset of received symbols. Indeed, it has been shown 63 that by including simple linear TPC techniques, the aforementioned 64 constructive interference can be exploited to boost the received power 65 of the information symbols in the multiple-input-single-output DL [2], 66 [18]. Here, we selectively apply this concept to a subset of received 67 symbols to enhance their power levels and convey the spatial symbol, 68 thus reusing interfering power in a power-efficient manner.

The remainder of this paper is organized as follows. Section II 70 introduces the proposed transmission scheme. Section III focuses 71 on the calculation of the computational complexity of the proposed 72 scheme, whereas in Section IV, we discuss the error probability of our 73 approach. Finally, Section V presents our numerical results, and our 74 conclusions are offered in Section VI.

\section{DUAL-LAYERED TRANSMISSION BY CONSTRUCTIVE INTERFERENCE}

\section{A. System Model}

Consider a MIMO system where the transmitter and the receiver 79 are equipped with $N_{t}$ and $N_{r}$ antennas, respectively. For simplicity, 80 unless stated otherwise, in this paper, we assume that the transmit 81 power budget is limited to $P=1$. For the case of the closed-form 82 TPCs in [1] and [2], it is required that $N_{t} \geq N_{r}$. The given channel is 83 modeled by

$$
\mathbf{r}=\mathbf{H t}+\mathbf{w}
$$

where $\mathbf{r}$ is the vector of received symbols in all RAs, and $\mathbf{H}$ is the 85 MIMO channel vector with elements $h_{m, n}$ representing the complex- 86 valued channel coefficient between the $n$th TA and the $m$ th RA. 87 Furthermore, $\mathbf{t}$ is the vector of precoded transmit symbols that will be 88 discussed in the following, and $\mathbf{w} \sim \mathcal{C N}\left(0, \sigma^{2} \mathbf{I}\right)$ is the additive white 89 Gaussian noise at the receiver, with $\mathcal{C N}\left(\mu, \sigma^{2}\right)$ denoting the circularly 90 symmetric complex Gaussian distribution associated with a mean of $\mu 91$ and a variance of $\sigma^{2}$. 


\section{B. Proposed DLT-CI}

94 The conventional DLT in [17] combines SMX with RSM where the 95 bandwidth efficiency (BE) of conventional SMX MIMO transmission 96 is strictly enhanced. This is achieved by encoding the spatial bits in the 97 RSM fashion in the received power domain, by selecting two distinct 98 nonzero power levels for the transmit supersymbols instead of the con99 ventional 'on-off' RSM transmission. This allows for having nonzero 100 elements throughout the received symbol vector and, therefore, a full 101 SMX transmission in the modulated signal domain. Here, we explore 102 the technique of forming the difference between the received power 103 levels for DLT by actively harvesting the constructive interference at 104 the receiver. This allows for 1) an improved BE of

$$
\epsilon=N_{r} \log _{2}(M)+\log _{2}\left(\begin{array}{c}
N_{r} \\
N_{a}
\end{array}\right)
$$

105 for DLT with an $M$-order modulation by transmission of the spatial 106 symbol, where $N_{a}$ denotes the number of higher-power received 107 symbols; for 2) enhanced power efficiency where the spatial symbol 108 is formed by the reuse of interference power instead of power allo109 cation; and for 3) an improved average error performance due to the 110 increased power levels of a subset of symbols by means of constructive 111 interference.

112 1) Transmitter: In [2], Masouros proposed a linear TPC that 113 carefully aligns interference so that it constructively contributes to 114 the desired signal power. In brief, the precoding matrix in [2] is 115 formed as

$$
\mathbf{T}_{c}=\mathbf{T R}_{\phi}
$$

116 where $\mathbf{T}=\mathbf{H}^{H}\left(\mathbf{H H}^{H}\right)^{-1}$, and $\mathbf{R}_{\phi}=\mathbf{R} \odot \mathbf{\Phi}$, with $\odot$ denoting 117 element-wise matrix multiplication and $\mathbf{R}_{\phi}$ representing the correla118 tion rotation $(\mathrm{CR})$ matrix that contains the elements of the channel 119 correlation matrix $\mathbf{R}=\mathbf{H H}^{H}$ rotated by the angle-only matrix $\boldsymbol{\Phi}$ 120 such that the resulting interference constructively aligns to the received 121 signal. To avoid repetition, see [2] for the details of the formation of $122 \mathbf{R}_{\phi}$, whereas here, we modify the above operation for our proposed 123 technique as detailed in the following. As an enhancement of the 124 conventional DLT in [17], we employ this concept here by first forming 125 the modulated symbol vector $\mathbf{b}_{m}=\left[b_{m_{1}}, b_{m_{2}}, \ldots, b_{m_{N_{r}}}\right]^{T}$ where, 126 as opposed to the DLT in [17], all symbols have the same power. 127 Here, $b_{m_{i}}, m_{i} \in\{1, \ldots, M\}$ is a symbol taken from an $M$-order 128 modulation alphabet that represents the transmitted waveform in the 129 baseband domain conveying $\log _{2}(M)$ bits.

130 We next form the power imbalance at the receiver by allowing 131 constructive interference for the $N_{a}$-out-of- $N_{r}$ RAs by appropriately 132 adapting the TPC in [2]. Explicitly, we modify the precoding matrix 133 of (3) to selectively allow constructive interference imposed only on 134 the $N_{a}$ "activated" antennas as a means of creating the required data135 dependent power difference. First, to ensure uniform power for the 136 desired symbol (excluding interference) across all RAs, we employ 137 a normalized version of the channel correlation matrix formulated as $138 \mathbf{Q}=\mathbf{R} \operatorname{diag}(\mathbf{R})^{-1}$ with ones along its diagonal. We use the operator $139 \operatorname{diag}(\mathbf{R})$ to denote the matrix that has the diagonal elements of $\mathbf{R}$ on 140 its diagonal and zeros elsewhere. The normalized CR matrix is then 141 formed as $\mathbf{Q}_{\phi}=\mathbf{Q} \odot \mathbf{\Phi}$. We then apply the precoding matrix

$$
\mathbf{T}^{k}=\mathbf{T Q}_{\phi}^{k}
$$

142 where $\mathbf{Q}_{\phi}^{k}=\left\{\mathbf{Q}_{\phi}\right\}_{k}$ is the selective CR matrix where the rows in set $143 k$ are taken from $\mathbf{Q}_{\phi}$, whereas the remaining rows are taken from the 144 identity matrix with size $N_{r}$. Finally, the transmit vector is formed as

$$
\mathbf{t}=\beta \mathbf{T}^{k} \mathbf{b}_{m}
$$

where $\beta=\sqrt{1 / \operatorname{tr}\left(\mathbf{T}^{k} \mathbf{T}^{k H}\right)}$ is the average power normalization fac- 145 tor. In the given equation, $k$ represents the index of the $N_{a}$ activated 146 RAs (the index of the high-power elements in the received vector) 147 conveying $\log _{2}\left(\begin{array}{l}N_{r} \\ N_{a}\end{array}\right)$ bits in the spatial domain. Matrix $\mathbf{T}^{k}$ can be 148 thought of as the combined precoding and spatial symbol matrix, 149 which only allows constructive interference to be imposed on the $N_{a} 150$ RAs as indicated by the spatial symbol $k$. From (1)-(5), the received 151 signal is given as

$$
\mathbf{r}=\beta \mathbf{Q}_{\phi}^{k} \mathbf{b}_{m}+\mathbf{w}
$$

where the dual-layered received supersymbol has been formed as 153 $\mathbf{s}_{m}^{k}=\beta \mathbf{Q}_{\phi}^{k} \mathbf{b}_{m}$. It can be seen that for the "inactive" RAs, we have 154

$$
r_{i}=\beta b_{m_{i}}+w_{i}, i \in \mathcal{L}
$$

where $\mathcal{L}$ is the set of "inactive" antennas. Clearly, for a normalized 155 modulation constellation, these symbols are received at power levels 156 of $P_{L}=\beta^{2}$. For the rest of the symbols, we have

$$
\begin{aligned}
r_{i} & =\beta \mathbf{q}_{\phi}^{i} \mathbf{b}_{m}+w_{i} \\
& =\beta b_{m_{i}}+\sum_{j \neq i}^{N_{r}} \mathbf{q}_{\phi}^{i, j} \mathbf{b}_{m_{j}}+w_{i}, i \in \mathcal{L}_{c}
\end{aligned}
$$

where $\mathbf{q}_{\phi}^{i}=\left[\mathbf{q}_{\phi}^{i, 1}, \mathbf{q}_{\phi}^{i, 2}, \ldots, \mathbf{q}_{\phi}^{i, N_{r}}\right]$ is the $i$ th row of $\mathbf{Q}_{\phi}^{k}$, and $\mathcal{L}_{c}$ is 158 the complementary set of $\mathcal{L}$, i.e., the set of $N_{a}$ "active" antennas. The 159 symbols in (8) are received at higher power levels due to constructive 160 interference [2]. Since for CR precoding, all interfering symbols are 161 constructively aligned to the symbol of interest, for the case of constant 162 envelope modulation, it can be seen that the received power levels obey 163

$$
P_{i}=\beta^{2}\left(1+\sum_{j \neq i}^{N_{r}}\left\|\mathbf{q}_{\phi}^{i, j}\right\|^{2}\right)>\beta^{2}=P_{L} .
$$

Clearly, this constructive interference is what creates the power level 164 separation between the RAs to form the spatial symbol $k$.

165

Remark: Note that a number of alternative precoders such as 166 [18]-[24] can be used in conjunction with the proposed approach to 167 accommodate constructive interference for the formation of the power 168 level separation required for DLT. To constrain the computational 169 complexity, here, we employ the low-complexity approach in [2], as 170 previously detailed.

2) Receiver: At the receiver side, explicit knowledge of the power 172 levels is not required, as long as the detector can distinguish between 173 the power levels. Hence, the receive processing is identical to that for 174 conventional DLT where, first, the $N_{a}$ "active" antenna indexes are 175 detected based on the $N_{a}$ highest received power levels among the 176 RAs-formed by constructive interference-according to

$$
\hat{k}=\arg \max _{j \in \mathcal{J}} \sum_{i=1}^{N_{a}}\left|r_{i, j}\right|^{2}
$$

where $\mathcal{J}$ denotes the set of symbols in the spatial domain, and the 178 modulated symbols at all RAs are detected as

$$
\hat{\mathbf{b}}_{m}=\arg \min _{n \in \mathcal{Q}}\left|\mathbf{r} / \beta-\mathbf{b}_{n}\right|^{2}
$$

where $\mathcal{Q}$ denotes the modulation constellation, and $b_{n}$ are the symbols 180 in the modulated symbol alphabet. 
TABLE I

COMPlEXITY FOR SMX, DLT, AND THE PRoposed DLT-CI SCHEME

\begin{tabular}{|l|c|}
\hline & Operations \\
\hline \hline Transmitter: & $N_{r}^{3}+N_{t} N_{r}+N_{t} N_{r} F$ \\
\hline ZF processing & $N_{r}^{3}+N_{t} N_{r}+\left(N_{t} N_{r}+N_{t} N_{r}^{2}\right) F$ \\
\hline Selective CR & $2 N_{a}\left(\begin{array}{c}N_{r} \\
N_{a}\end{array}\right) F$ \\
\hline Receiver: & $N_{r} M F$ \\
\hline Spatial detection & $N_{r}^{3}+N_{t} N_{r}+N_{r}\left(N_{t}+M\right) F$ \\
\hline Demodulation & $N_{r}^{3}+N_{t} N_{r}+$ \\
\hline \hline SMX Total & $N_{t} N_{r}+\left[N_{r}\left(N_{t}+M\right)+2 N_{a}\left(\begin{array}{c}N_{r} \\
N_{a}\end{array}\right)\right] F$ \\
\hline DLT Total & $\left.N_{r}^{3}+N_{r}\left(N_{t}+N_{t} N_{r}+M\right)+2 N_{a}\left(\begin{array}{c}N_{r} \\
N_{a}\end{array}\right)\right] F$ \\
\hline DLT-CI Total & \\
\hline
\end{tabular}

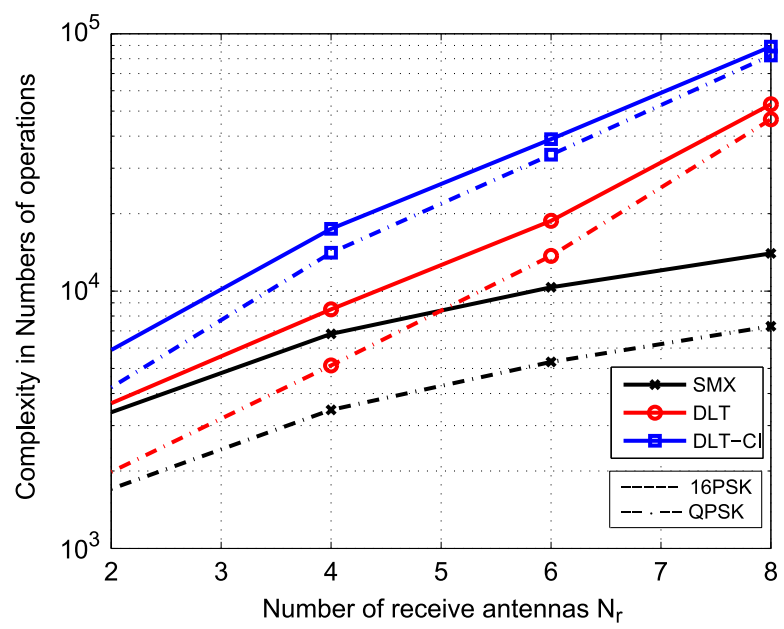

Fig. 1. Complexity versus $N_{r}$ for $N_{t}=8$ and $N_{a}=N_{r} / 2$ with SMX, DLT, and DLT-CI.

\section{Computational CompleXity}

183 Here, we compare the computational complexity of SMX, DLT, and 184 DLT-CI. First, Table I summarizes the computational complexity of 185 each of the techniques, taking into account the dominant operations 186 at the transmitter and the receiver. We assume a quasi-static channel, 187 which is constant for a frame length of $F$ supersymbols. For SMX 188 and DLT, the zero-forcing precoding at the transmitter involves the 189 inversion of the channel matrix that involves a number of $N_{r}^{3}+$ $190 N_{t} N_{r}$ operations and the multiplication with the supersymbol vector 191 involving an additional $N_{t} N_{r}$ operations for the $F$ supersymbols 192 of the transmission frame. The selective CR of DLT-CI involves 193 the additional multiplication of the precoding matrix with $\mathbf{Q}_{\phi}^{k}$ at 194 every symbol period, with complexity of $N_{t} N_{r}^{2}$. At the receiver, all 195 techniques require a demodulation stage that involves $M$ comparisons 196 for $M$-order modulation for each of the $N_{r}$ RAs. The DLT and 197 DLT-CI require an additional stage for the detection of the spatial 198 symbol, which, from (10), involves $N_{a}$ complex multiplications and $199 N_{a}$ complex additions for each antenna combination out of the $\left(\begin{array}{l}N_{r} \\ N_{a}\end{array}\right)$ 200 combinations in total.

201 Fig. 1 shows the complexity of SMX, DLT, and the proposed 202 DLT-CI for a system with $N_{t}=8$ TAs and increasing numbers of 203 RAs $N_{r}$, with $N_{a}=N_{r} / 2$. For reference, we have assumed a Long204 Term Evolution (LTE) Type-2 time-division duplexing (TDD) frame 205 structure for which $F=70$, as detailed in [17]. A slow-fading channel 206 is assumed where the channel remains constant for the duration of 207 the frame. It can be seen that the proposed DLT-CI has increased complexity compared with DLT. However, it will be shown in the 208 following results that the improved performance for DLT-CI is worth 209 the added complexity.

\section{ERRor ProbabiLITy}

The error probability of the proposed scheme can be described by 212 means of the pairwise error probability (PEP) $\mathcal{P}\left(\mathbf{s}_{m}^{k} \rightarrow \mathbf{s}_{n}^{l}\right)$. By the 213 use of the union bound, the average bit error probability $P_{e}$ can be 214 expressed as [13]

$$
P_{e} \leq \frac{1}{b} E\left\{\sum_{\mathbf{s}_{m}^{k} \in \mathcal{B} \mathbf{s}_{n}^{l} \in \mathcal{B} \neq \mathbf{s}_{m}^{k}} d\left(\mathbf{s}_{m}^{k}, \mathbf{s}_{n}^{l}\right) \mathcal{P}\left(\mathbf{s}_{m}^{k} \rightarrow \mathbf{s}_{n}^{l}\right)\right\}
$$

where $d\left(\mathbf{s}_{m}^{k}, \mathbf{s}_{n}^{l}\right)$ is the Hamming distance between the bit representa- 216 tions of the symbols $\mathbf{s}_{m}^{k}, \mathbf{s}_{n}^{l}$, and $\mathcal{B}$ is the supersymbol constellation 217 defined as the union of the spatial-domain constellation and of the 218 classic modulation constellation. The PEP can further be decomposed 219 into the PEP for the spatial symbol $\mathcal{P}\left(s_{m_{i}}^{k} \rightarrow s_{m_{i}}^{l}\right)$ and the PEP 220 $\mathcal{P}\left(s_{m_{i}}^{k} \rightarrow s_{n_{i}}^{k}\right)$ of the modulated symbol. These obey the following 221 lemmas.

Lemma 1: The PEP of the spatial symbol for the DLT-CI transmis- 223 sion obeys

$$
\mathcal{P}\left(s_{m_{i}}^{k} \rightarrow s_{m_{i}}^{l}\right)=Q\left(\frac{\beta}{\sqrt{2 \sigma^{2}}} \cdot \frac{\sqrt{P_{i}}-\sqrt{P_{L}}}{2}\right)
$$

where $Q($.$) denotes the Gaussian Q-function.$

Lemma 2: The PEP for the $M$-order phase-shift keying ( $M$-PSK) 226 modulated symbol, which is the focus of this work, follows:

$$
\mathcal{P}\left(s_{m_{i}}^{k} \rightarrow s_{n_{i}}^{k}\right)=Q\left(\beta \sqrt{\frac{P_{i}}{2 \sigma^{2}} \log _{2}(M) \sin \frac{\pi}{M}}\right) .
$$

Both the above expressions can be straightforwardly derived by adapt- 228 ing the methodology introduced in [17] for the proposed scenario. It is 229 the PEP in (14) that is enhanced for the proposed scheme by allowing 230 constructive interference to increase $P_{i}$. The tightness of the above- 231 described bound is validated in Section V.

\section{NumericAl RESUlts}

To evaluate the benefits of the proposed technique, this section 234 presents Monte Carlo simulations of the proposed DLT-CI in compar- 235 ison to conventional approaches. As the superiority of conventional 236 DLT over the most relevant SM and SMX approaches was thoroughly 237 validated in [17] and to limit the congestion in the following graphs, 238 here, we only use conventional DLT and SMX as a reference for 239 comparison. The channel impulse response is assumed to be perfectly 240 known at the transmitter for all techniques. Without loss of generality, 241 unless stated otherwise, we assume that the transmit power is restricted 242 to $P=1$. MIMO systems with up to eight TAs employing quaternary 243 phase-shift keying (QPSK), 8-PSK, and 16-PSK modulation are ex- 244 plored, albeit it is plausible that the benefits of the proposed technique 245 extend to larger-scale systems and higher-order modulation. For DLT 246 and DLT-CI, we focus on the case $N_{a}=N_{r} / 2$, which provides the 247 highest BE [17].

In Figs. 2 and 3, we show the BER with increasing signal-to- 249 noise ratio (SNR) for QPSK and 8-PSK, respectively. To complete 250 our comparisons, for both scenarios in the figure, we also show the 251 cases where the symbol modulation order used for SMX is increased 252 for some of the spatial streams to achieve the same BE values of 253 $\epsilon=10$ and $\epsilon=14$ with the proposed DLT, for QPSK and 8-PSK, 254 respectively. The figures also show the theoretical bound of (13) on 255 


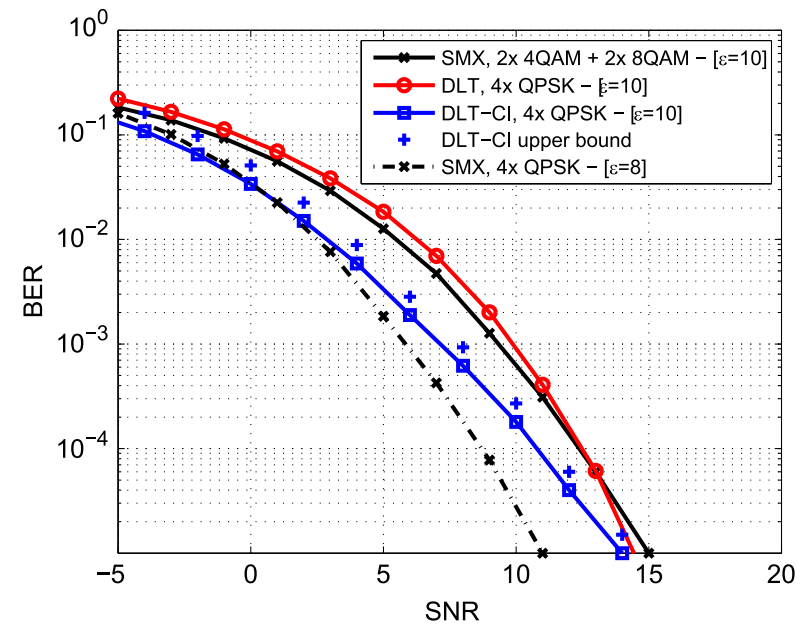

Fig. 2. BER versus SNR for a $(8 \times 4)$ MIMO with SMX, DLT, and DLT-CI; QPSK modulation.

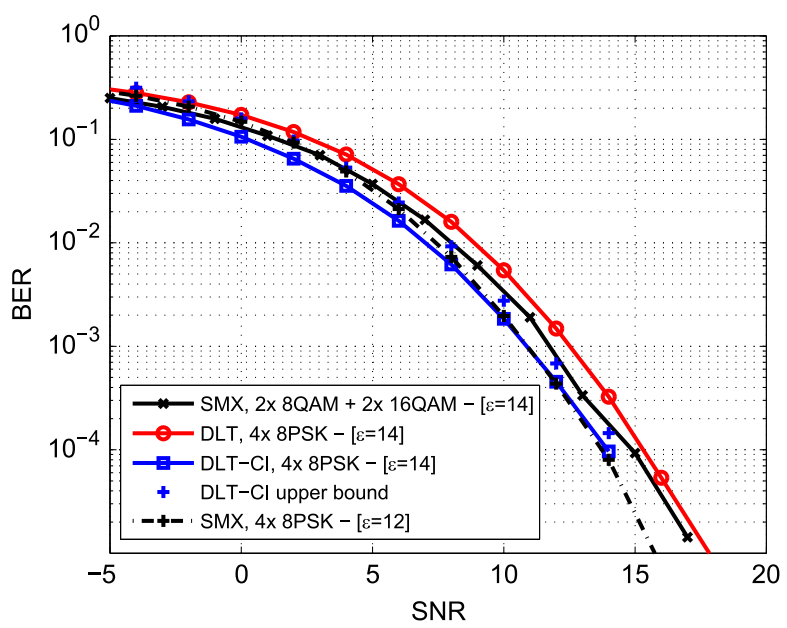

Fig. 3. BER versus SNR for a $(8 \times 4)$ MIMO with SMX, DLT, and DLT-CI; 8-PSK modulation.

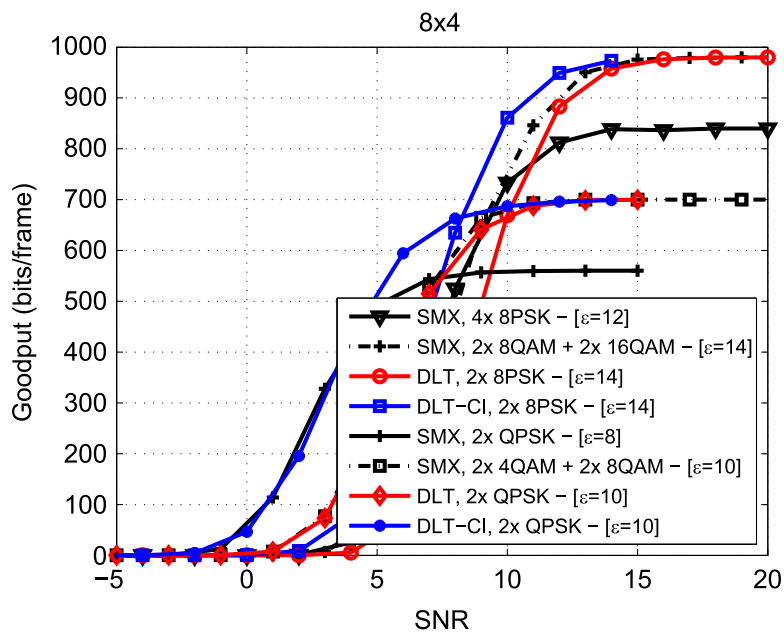

Fig. 4. Throughput versus SNR for a $(8 \times 4)$ MIMO with SMX, DLT, and DLT-CI.

256 the error probability, which closely matches our simulation results in 257 both cases. Clearly, the DLT scheme has an inferior BER performance 258 compared with SMX due to the additional spatial streams, which is the

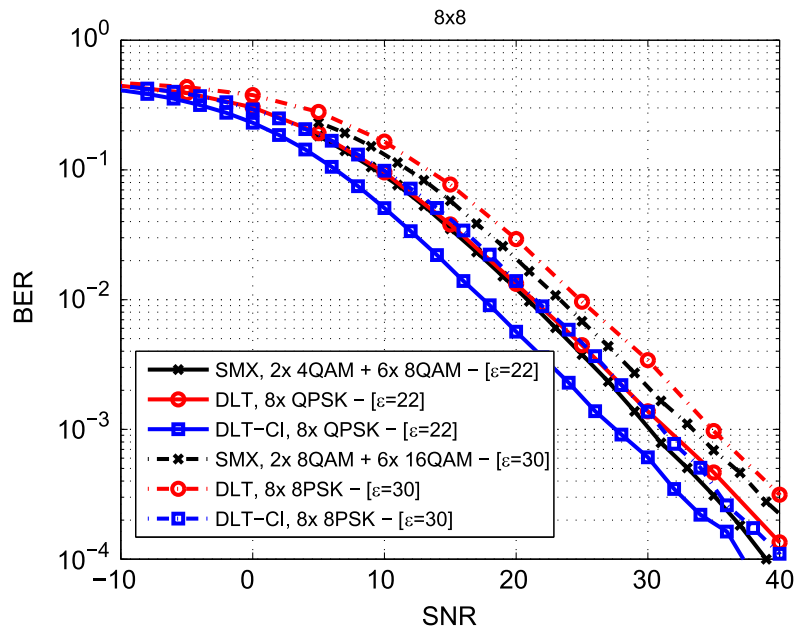

Fig. 5. BER versus SNR for a $(8 \times 8)$ MIMO with SMX, DLT, and DLT-CI.

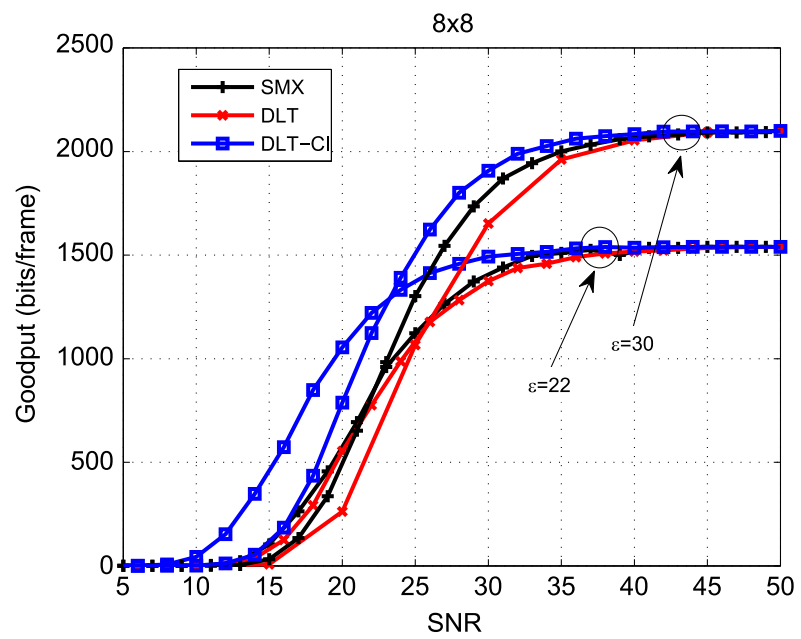

Fig. 6. Throughput versus SNR for a $(8 \times 8)$ MIMO with SMX, DLT, and DLT-CI.

price paid for its improved BE. DLT-CI outperforms both SMX and 259 DLT as an explicit benefit of the constructive interference exploited 260 as useful signal power, both in the modulated symbol detection and 261 in the formation of the different power levels employed for the spatial 262 symbol transmission. The improved BE of DLT-CI is demonstrated in 263 Fig. 4,where goodput versus SNR is depicted for the same $(8 \times 4) 264$ MIMO scenario. The goodput here is defined as $R=\epsilon F\left(1-P_{e}\right)^{F}, 265$ where $P_{e}$ is the bit error probability [17]. For reference, we have 266 assumed an LTE Type-2 TDD frame structure for which we have 267 $F=70$, as detailed in [17]. Clearly, DLT-CI provides the best goodput 268 performance among the schemes explored.

Our performance comparison is extended to the $(8 \times 8)$ MIMO 270 system in Figs. 5 and 6. The BER performance with increasing SNR is 271 shown in Fig. 5 for the $(8 \times 8)$ MIMO system where it can be seen that 272 DLT-CI outperforms both SMX and DLT. Fig. 6 shows the goodput 273 with increasing SNR, where, again, it can be observed that DLT-CI 274 provides the best goodput.

\section{CONCLUSION}

An enhanced dual-layered DL transmission scheme has been pro- 277 posed, which combines traditional MIMO SMX with RSM. The 278 proposed scheme improves upon conventional DLT by allowing con- 279 structive interference to carry spatial information, as opposed to the 280 
281 fixed power-level split of the conventional DLT in [17]. Our results show 282 that by allowing constructive interference to separate the power levels 283 and convey the spatial symbol, the proposed DLT-CI improves the BE 284 of SMX while, at the same time, the increased power levels of the sub285 set of symbols improve the average error performance of the system.

\section{REFERENCES}

1] C. B. Peel, B. M. Hochwald, and A. L. Swindlehurst, "A vectorperturbation technique for near-capacity multiantenna multiuser communication-Part I: Channel inversion and regularization," IEEE Trans. Commun., vol. 53, no. 1, pp. 195-202, Jan. 2005.

[2] C. Masouros, "Correlation rotation linear precoding for MIMO broadcast communications," IEEE Trans. Signal Process., vol. 59, no. 1, pp. 252-262, Jan. 2011.

[3] R. Mesleh, H. Haas, S. Sinanovic, C. W. Ahn, and S. Yun, "Spatial Modulation," IEEE Trans. Veh. Technol., vol. 57, no. 4, pp. 2228-2241, Jul. 2008.

[4] M. Di Renzo, H. Haas, A. Ghrayeb, S. Sugiura, and L. Hanzo, "Spatial modulation for generalized MIMO: Challenges, opportunities, and implementation," Proc. IEEE, vol. 102, no. 1, pp.56-103, Jan. 2014.

[5] Y. Ping, M. Di Renzo, X. Yue, L. Shaoqian, and L. Hanzo, "Design guidelines for spatial modulation," IEEE Commun. Surveys Tuts., vol. 17, no. 1, pp. 6-26, 1st Quart. 2015.

[6] M. Di Renzo and H. Haas, "On transmit diversity for spatial modulation MIMO: Impact of spatial constellation diagram and shaping filters at the transmitter," IEEE Trans. Veh. Technol., vol. 62, no. 6, pp. 2507-2531, Jul. 2013.

[7] P. Yang et al., "Star-QAM signaling constellations for spatial modulation," IEEE Trans. Veh. Technol., vol. 63, no. 8, pp. 3741-3749, Oct. 2014.

[8] S. Sugiura and L. Hanzo, "On the joint optimization of dispersion matrices and constellations for near-capacity irregular precoded space-time shift keying," IEEE Trans. Wireless Commun., vol. 12, no. 1, pp. 380-387, Jan. 2013.

[9] M. Maleki, H. Bahrami, S. Beygi, M. Kafashan, and N. H. Tran, "Space modulation with CSI: Constellation design and performance evaluation," IEEE Trans. Veh. Technol., vol. 62, no. 4, pp. 1623-1634, May 2013.

10] X. Guan, Y. Cai, and W. Yang, "On the mutual information and precoding for spatial modulation with finite alphabet," IEEE Wireless Commun. Lett., vol. 2, no. 4, pp. 383-386, Aug. 2013.
[11] C. Masouros, "Improving the diversity of spatial modulation in MISO 320 channels by phase alignment," IEEE Commun. Lett., vol. 18, no. 5, 321 pp. 729-732, May 2014.

[12] M. Di Renzo and H. Haas, "Improving the performance of Space Shift 323 Keying (SSK) modulation via opportunistic power allocation," IEEE 324 Commun. Lett., vol. 14, no. 6, pp. 500-502, Jun. 2010.

[13] C. Masouros and L. Hanzo, "Constellation-randomization achieves 326 transmit diversity for single-RF spatial modulation," IEEE Trans. Veh. 327 Technol., to be published.

[14] A. Garcia, C. Masouros, and L. Hanzo, "Pre-scaling optimization for 329 space shift keying based on semidefinite relaxation," IEEE Trans. Com- 330 mun., vol. 63, no. 11, pp. 4231-4243, Nov. 2015.

331

[15] R. Zhang, L. Yang, and L. Hanzo, "Generalised pre-coding aided spa- 332 tial modulation," IEEE Trans. Wireless Commun., vol. 12, no. 11, 333 pp. 5434-5443, Nov. 2013.

[16] L.-L. Yang, "Transmitter preprocessing aided spatial modulation 335 for multiple-input multiple-output systems," in Proc. IEEE 73rd 336 VTC-Spring, May 15-18, 2011, pp. 1-5.

[17] C. Masouros and L. Hanzo, "Dual layered downlink MIMO transmis- 338 sion for increased bandwidth efficiency," IEEE Trans. Commun., to be 339 published.

[18] C. Masouros and E. Alsusa, "Soft linear precoding for the downlink of 341 DS/CDMA communication systems," IEEE Trans. Veh. Technol., vol. 59, 342 no. 1 , pp. 203-215, Jan. 2010.

[19] C. Masouros and E. Alsusa, "Dynamic linear precoding for the exploita- 344 tion of known interference in MIMO broadcast systems," IEEE Trans. 345 Wireless Commun., vol. 8, no. 3, pp. 1396-1404, Mar. 2009.

346

[20] C. Masouros, T. Ratnarajah, M. Sellathurai, C. Papadias, and A. Shukla, 347 "Known interference in wireless communications: A limiting factor or a 348 potential source of green signal power?" IEEE Commun. Mag., vol. 51, 349 no. 10, pp. 162-171, Oct. 2013.

[21] G. Zheng et al., "Rethinking the role of interference in wireless networks," 351 IEEE Commun. Mag., vol. 52, no. 11, pp. 152-158, Nov. 2014.

[22] C. Masouros and E. Alsusa, "Two-stage transmitter precoding based on 353 data-driven code hopping and partial zero forcing beamforming for MC- 354 CDMA communications," IEEE Trans. Wireless Commun., vol. 8, no. 7, 355 pp. 3634-3645, Jul. 2009.

[23] C. Masouros and G. Zheng, "Exploiting known interference as green sig- 357 nal power for downlink beamforming optimization," IEEE Trans. Signal 358 Process., vol. 63, no. 14, pp. 3668-3680, Jul. 2015.

[24] C. Masouros, M. Sellathurai, and T. Ratnarajah, "Vector perturbation 360 based on symbol scaling for limited feedback MISO downlinks," IEEE 361 Trans. Signal. Process., vol. 62, no. 3, pp. 562-571, Feb. 1, 2014. 


\section{AUTHOR QUERIES}

\section{AUTHOR PLEASE ANSWER ALL QUERIES}

AQ1 = Please provide the complete current affiliation of author C. Masouros and check if the provided affiliation of author L. Hanzo is correct.

AQ2 = Uncited Figure 4. Figure 6 was changed to Figure 4. Please check if correct.

AQ3 = Please provide publication update in Ref. [13].

AQ4 = Please provide publication update in Ref. [17].

\section{END OF ALL QUERIES}




\section{Correspondence}

\section{Constructive Interference as an Information Carrier by Dual-Layered MIMO Transmission}

3

4

\author{
Christos Masouros, Senior Member, IEEE, and \\ Lajos Hanzo, Fellow, IEEE
}

\begin{abstract}
5 Abstract-We propose a bandwidth-efficient transmission scheme for 6 multiple-input-multiple-output point-to-point and downlink channels. 7 The bandwidth efficiency (BE) of spatial multiplexing (SMX) is improved 8 by implicitly encoding information in the spatial domain based on the exis9 tence of constructive interference in the received symbols, which creates a 10 differentiation in the symbol power. Explicitly, the combination of symbols 11 received at a higher power level carries implicit information in the spatial 12 domain in the same manner as that the combination of nonzero elements in 13 the received symbol vector carries information for receive-antenna-based 14 spatial modulation (RSM). The nonzero power throughout the received 15 symbol vector for the proposed technique allows a full SMX underlying 16 transmission, with the BE enhancement brought by the spatial symbol. 17 Our simulation results demonstrate both significant BE gains and error 18 probability reduction for our approach over the conventional SMX and 19 RSM schemes.
\end{abstract}

20 Index Terms-Multiple-input multiple-output (MIMO), precoding, spa21 tial modulation (RSM), spatial multiplexing (SMX).

\section{INTRODUCTION}

23 Multiple-input multiple-output (MIMO) systems have been shown 24 to improve the capacity of the wireless channel by means of spatial 25 multiplexing (SMX). Transmit precoding (TPC) schemes introduced 26 for multiuser downlink (DL) transmission improve both the power 27 efficiency and cost of mobile stations by shifting the signal processing 28 complexity to the base stations. From the wide range of linear and 29 nonlinear TPC schemes found in the literature, here, we focus our 30 attention on the family of closed-form linear TPC schemes based on 31 channel inversion [1], [2], which pose low computational complexity. 32 More recently, spatial modulation (SM) has been explored as a means 33 of implicitly encoding information in the index of the specific transmit 34 antenna (TA) activated for the transmission of the modulated symbols, 35 which offers a low-complexity design alternative [3]. Its central bene36 fits include the absence of interantenna interference and the fact that, 37 in contrast to SMX, it only requires a subset (down to one) of radio38 frequency chains compared with SMX. Early work has focused on the 39 design of receiver algorithms for minimizing the bit error ratio (BER) 40 of SM at low complexity [3]-[5].

41 In addition to receive processing, recent work has also proposed 42 constellation shaping for SM [6]-[14]. Specifically, the contributions 43 on this topic have focused on three main directions: 1) shaping and

Manuscript received June 20, 2015; revised November 3, 2015; accepted February 8, 2016. This work was supported in part by the U.K. Royal Academy of Engineering and in part by the Engineering and Physical Sciences Research Council through Project EP/M014150/1. The review of this paper was coordinated by Prof. W. A. Krzymień.

C. Masouros is with London WC1E 7JE, U.K. (e-mail: c.masouros@ucl.ac. uk).

L. Hanzo is with the School of Electronics and Computer Science, University of Southampton, Southampton SO17 1BJ, U.K. (e-mail: 1h@ecs.soton.ac.uk).

Color versions of one or more of the figures in this paper are available online at http://ieeexplore.ieee.org.

Digital Object Identifier 10.1109/TVT.2016.2528506 optimization of the spatial constellation, i.e., the legitimate sets of 44 activated TAs [6]; 2) modulation constellation shaping [7]-[9] for the 45 SM transmission where the constellation of the classically modulated 46 bits is optimized; and 3) joint spatial and modulation constellation 47 shaping, in the form of optimizing the received constellation [10]-[14]. 48 Closely related treatises have been focused on applying SM to the 49 receive antennas (RAs) of the communication link, forming the RA- 50 based spatial modulation (RSM) regime [15], [16]. By means of 51 precoding at the transmitter, this regime aims at transmitting to a 52 reduced a subset of RAs that receive information symbols, whereas the 53 rest of the antennas receive only noise. A dual-layered transmission 54 (DLT) scheme was proposed in [17], where the spatial symbol is 55 conveyed, not by transmitting a combination of symbols and zeros 56 but by assigning a pair of power levels $\left\{P_{1}, P_{2}\right\}$ to the received 57 symbols, with the combination of power levels detected at the receiver 58 representing a spatial symbol.

Here, we explore a power-efficient alternative, where the distinction 60 of the power levels in DLT is no longer formed by the aforementioned 61 direct power allocation but rather by allowing the constructive interfer- 62 ence to form a subset of received symbols. Indeed, it has been shown 63 that by including simple linear TPC techniques, the aforementioned 64 constructive interference can be exploited to boost the received power 65 of the information symbols in the multiple-input-single-output DL [2], 66 [18]. Here, we selectively apply this concept to a subset of received 67 symbols to enhance their power levels and convey the spatial symbol, 68 thus reusing interfering power in a power-efficient manner.

The remainder of this paper is organized as follows. Section II 70 introduces the proposed transmission scheme. Section III focuses 71 on the calculation of the computational complexity of the proposed 72 scheme, whereas in Section IV, we discuss the error probability of our 73 approach. Finally, Section V presents our numerical results, and our 74 conclusions are offered in Section VI.

\section{DUAL-LAYERED TRANSMISSION BY} CONSTRUCTIVE INTERFERENCE

\section{A. System Model}

Consider a MIMO system where the transmitter and the receiver 79 are equipped with $N_{t}$ and $N_{r}$ antennas, respectively. For simplicity, 80 unless stated otherwise, in this paper, we assume that the transmit 81 power budget is limited to $P=1$. For the case of the closed-form 82 TPCs in [1] and [2], it is required that $N_{t} \geq N_{r}$. The given channel is 83 modeled by

$$
\mathbf{r}=\mathbf{H t}+\mathbf{w}
$$

where $\mathbf{r}$ is the vector of received symbols in all RAs, and $\mathbf{H}$ is the 85 MIMO channel vector with elements $h_{m, n}$ representing the complex- 86 valued channel coefficient between the $n$th TA and the $m$ th RA. 87 Furthermore, $\mathbf{t}$ is the vector of precoded transmit symbols that will be 88 discussed in the following, and $\mathbf{w} \sim \mathcal{C N}\left(0, \sigma^{2} \mathbf{I}\right)$ is the additive white 89 Gaussian noise at the receiver, with $\mathcal{C N}\left(\mu, \sigma^{2}\right)$ denoting the circularly 90 symmetric complex Gaussian distribution associated with a mean of $\mu 91$ and a variance of $\sigma^{2}$. 


\section{B. Proposed DLT-CI}

94 The conventional DLT in [17] combines SMX with RSM where the 95 bandwidth efficiency (BE) of conventional SMX MIMO transmission 96 is strictly enhanced. This is achieved by encoding the spatial bits in the 97 RSM fashion in the received power domain, by selecting two distinct 98 nonzero power levels for the transmit supersymbols instead of the con99 ventional 'on-off' RSM transmission. This allows for having nonzero 100 elements throughout the received symbol vector and, therefore, a full 101 SMX transmission in the modulated signal domain. Here, we explore 102 the technique of forming the difference between the received power 103 levels for DLT by actively harvesting the constructive interference at 104 the receiver. This allows for 1) an improved BE of

$$
\epsilon=N_{r} \log _{2}(M)+\log _{2}\left(\begin{array}{c}
N_{r} \\
N_{a}
\end{array}\right)
$$

105 for DLT with an $M$-order modulation by transmission of the spatial 106 symbol, where $N_{a}$ denotes the number of higher-power received 107 symbols; for 2) enhanced power efficiency where the spatial symbol 108 is formed by the reuse of interference power instead of power allo109 cation; and for 3) an improved average error performance due to the 110 increased power levels of a subset of symbols by means of constructive 111 interference.

112 1) Transmitter: In [2], Masouros proposed a linear TPC that 113 carefully aligns interference so that it constructively contributes to 114 the desired signal power. In brief, the precoding matrix in [2] is 115 formed as

$$
\mathbf{T}_{c}=\mathbf{T R}_{\phi}
$$

116 where $\mathbf{T}=\mathbf{H}^{H}\left(\mathbf{H} \mathbf{H}^{H}\right)^{-1}$, and $\mathbf{R}_{\phi}=\mathbf{R} \odot \mathbf{\Phi}$, with $\odot$ denoting 117 element-wise matrix multiplication and $\mathbf{R}_{\phi}$ representing the correla118 tion rotation $(\mathrm{CR})$ matrix that contains the elements of the channel 119 correlation matrix $\mathbf{R}=\mathbf{H H}^{H}$ rotated by the angle-only matrix $\boldsymbol{\Phi}$ 120 such that the resulting interference constructively aligns to the received 121 signal. To avoid repetition, see [2] for the details of the formation of $122 \mathbf{R}_{\phi}$, whereas here, we modify the above operation for our proposed 123 technique as detailed in the following. As an enhancement of the 124 conventional DLT in [17], we employ this concept here by first forming 125 the modulated symbol vector $\mathbf{b}_{m}=\left[b_{m_{1}}, b_{m_{2}}, \ldots, b_{m_{N_{r}}}\right]^{T}$ where, 126 as opposed to the DLT in [17], all symbols have the same power. 127 Here, $b_{m_{i}}, m_{i} \in\{1, \ldots, M\}$ is a symbol taken from an $M$-order 128 modulation alphabet that represents the transmitted waveform in the 129 baseband domain conveying $\log _{2}(M)$ bits.

130 We next form the power imbalance at the receiver by allowing 131 constructive interference for the $N_{a}$-out-of- $N_{r}$ RAs by appropriately 132 adapting the TPC in [2]. Explicitly, we modify the precoding matrix 133 of (3) to selectively allow constructive interference imposed only on 134 the $N_{a}$ "activated" antennas as a means of creating the required data135 dependent power difference. First, to ensure uniform power for the 136 desired symbol (excluding interference) across all RAs, we employ 137 a normalized version of the channel correlation matrix formulated as $138 \mathbf{Q}=\mathbf{R} \operatorname{diag}(\mathbf{R})^{-1}$ with ones along its diagonal. We use the operator $139 \operatorname{diag}(\mathbf{R})$ to denote the matrix that has the diagonal elements of $\mathbf{R}$ on 140 its diagonal and zeros elsewhere. The normalized CR matrix is then 141 formed as $\mathbf{Q}_{\phi}=\mathbf{Q} \odot \boldsymbol{\Phi}$. We then apply the precoding matrix

$$
\mathbf{T}^{k}=\mathbf{T} \mathbf{Q}_{\phi}^{k}
$$

142 where $\mathbf{Q}_{\phi}^{k}=\left\{\mathbf{Q}_{\phi}\right\}_{k}$ is the selective CR matrix where the rows in set $143 k$ are taken from $\mathbf{Q}_{\phi}$, whereas the remaining rows are taken from the 144 identity matrix with size $N_{r}$. Finally, the transmit vector is formed as

$$
\mathbf{t}=\beta \mathbf{T}^{k} \mathbf{b}_{m}
$$

where $\beta=\sqrt{1 / \operatorname{tr}\left(\mathbf{T}^{k} \mathbf{T}^{k H}\right)}$ is the average power normalization fac- 145 tor. In the given equation, $k$ represents the index of the $N_{a}$ activated 146 RAs (the index of the high-power elements in the received vector) 147 conveying $\log _{2}\left(\begin{array}{l}N_{r} \\ N_{a}\end{array}\right)$ bits in the spatial domain. Matrix $\mathbf{T}^{k}$ can be 148 thought of as the combined precoding and spatial symbol matrix, 149 which only allows constructive interference to be imposed on the $N_{a} 150$ RAs as indicated by the spatial symbol $k$. From (1)-(5), the received 151 signal is given as

$$
\mathbf{r}=\beta \mathbf{Q}_{\phi}^{k} \mathbf{b}_{m}+\mathbf{w}
$$

where the dual-layered received supersymbol has been formed as 153 $\mathbf{s}_{m}^{k}=\beta \mathbf{Q}_{\phi}^{k} \mathbf{b}_{m}$. It can be seen that for the "inactive" RAs, we have 154

$$
r_{i}=\beta b_{m_{i}}+w_{i}, i \in \mathcal{L}
$$

where $\mathcal{L}$ is the set of "inactive" antennas. Clearly, for a normalized 155 modulation constellation, these symbols are received at power levels 156 of $P_{L}=\beta^{2}$. For the rest of the symbols, we have

$$
\begin{aligned}
r_{i} & =\beta \mathbf{q}_{\phi}^{i} \mathbf{b}_{m}+w_{i} \\
& =\beta b_{m_{i}}+\sum_{j \neq i}^{N_{r}} \mathbf{q}_{\phi}^{i, j} \mathbf{b}_{m_{j}}+w_{i}, i \in \mathcal{L}_{c}
\end{aligned}
$$

where $\mathbf{q}_{\phi}^{i}=\left[\mathbf{q}_{\phi}^{i, 1}, \mathbf{q}_{\phi}^{i, 2}, \ldots, \mathbf{q}_{\phi}^{i, N_{r}}\right]$ is the $i$ th row of $\mathbf{Q}_{\phi}^{k}$, and $\mathcal{L}_{c}$ is 158 the complementary set of $\mathcal{L}$, i.e., the set of $N_{a}$ "active" antennas. The 159 symbols in (8) are received at higher power levels due to constructive 160 interference [2]. Since for CR precoding, all interfering symbols are 161 constructively aligned to the symbol of interest, for the case of constant 162 envelope modulation, it can be seen that the received power levels obey 163

$$
P_{i}=\beta^{2}\left(1+\sum_{j \neq i}^{N_{r}}\left\|\mathbf{q}_{\phi}^{i, j}\right\|^{2}\right)>\beta^{2}=P_{L} .
$$

Clearly, this constructive interference is what creates the power level 164 separation between the RAs to form the spatial symbol $k$.

165

Remark: Note that a number of alternative precoders such as 166 [18]-[24] can be used in conjunction with the proposed approach to 167 accommodate constructive interference for the formation of the power 168 level separation required for DLT. To constrain the computational 169 complexity, here, we employ the low-complexity approach in [2], as 170 previously detailed.

2) Receiver: At the receiver side, explicit knowledge of the power 172 levels is not required, as long as the detector can distinguish between 173 the power levels. Hence, the receive processing is identical to that for 174 conventional DLT where, first, the $N_{a}$ "active" antenna indexes are 175 detected based on the $N_{a}$ highest received power levels among the 176 RAs-formed by constructive interference-according to

$$
\hat{k}=\arg \max _{j \in \mathcal{J}} \sum_{i=1}^{N_{a}}\left|r_{i, j}\right|^{2}
$$

where $\mathcal{J}$ denotes the set of symbols in the spatial domain, and the 178 modulated symbols at all RAs are detected as

$$
\hat{\mathbf{b}}_{m}=\arg \min _{n \in \mathcal{Q}}\left|\mathbf{r} / \beta-\mathbf{b}_{n}\right|^{2}
$$

where $\mathcal{Q}$ denotes the modulation constellation, and $b_{n}$ are the symbols 180 in the modulated symbol alphabet. 
TABLE I

COMPLEXITY FOR SMX, DLT, AND THE PROPOSED DLT-CI SCHEME

\begin{tabular}{|l|c|}
\hline & Operations \\
\hline \hline Transmitter: & $N_{r}^{3}+N_{t} N_{r}+N_{t} N_{r} F$ \\
\hline ZF processing & $N_{r}^{3}+N_{t} N_{r}+\left(N_{t} N_{r}+N_{t} N_{r}^{2}\right) F$ \\
\hline Selective CR & $2 N_{a}\left(\begin{array}{c}N_{r} \\
N_{a}\end{array}\right) F$ \\
\hline Receiver: & $N_{r} M F$ \\
\hline Spatial detection & $N_{r}^{3}+N_{t} N_{r}+N_{r}\left(N_{t}+M\right) F$ \\
\hline Demodulation & $N_{r}^{3}+N_{t} N_{r}+$ \\
\hline \hline SMX Total & $N_{t} N_{r}+\left[N_{r}\left(N_{t}+M\right)+2 N_{a}\left(\begin{array}{l}N_{r} \\
N_{a}\end{array}\right)\right] F$ \\
\hline DLT Total & {$\left[N_{r}\left(N_{t}+N_{t} N_{r}+M\right)+2 N_{a}\left(\begin{array}{l}N_{r} \\
N_{a}\end{array}\right)\right] F$} \\
\hline DLT-CI Total & \\
\hline
\end{tabular}

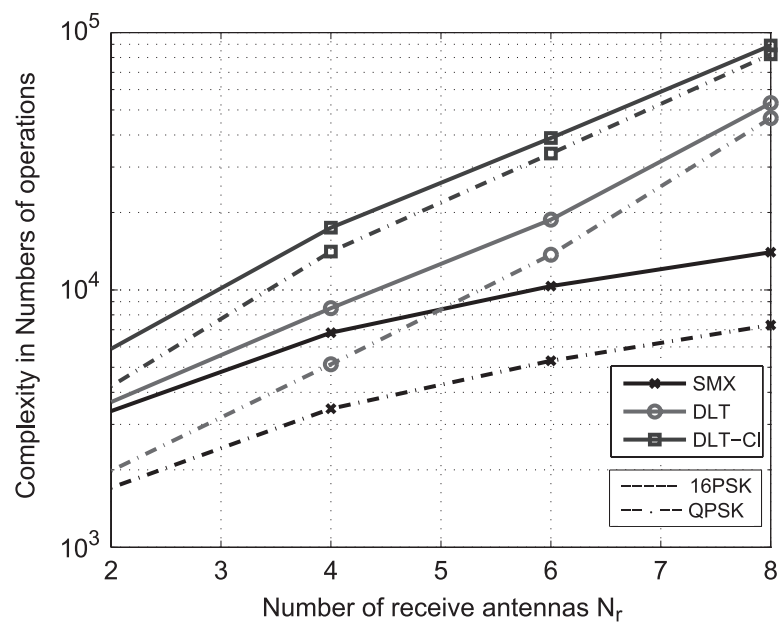

Fig. 1. Complexity versus $N_{r}$ for $N_{t}=8$ and $N_{a}=N_{r} / 2$ with SMX, DLT, and DLT-CI.

\section{Computational Complexity}

183 Here, we compare the computational complexity of SMX, DLT, and 184 DLT-CI. First, Table I summarizes the computational complexity of 185 each of the techniques, taking into account the dominant operations 186 at the transmitter and the receiver. We assume a quasi-static channel, 187 which is constant for a frame length of $F$ supersymbols. For SMX 188 and DLT, the zero-forcing precoding at the transmitter involves the 189 inversion of the channel matrix that involves a number of $N_{r}^{3}+$ $190 N_{t} N_{r}$ operations and the multiplication with the supersymbol vector 191 involving an additional $N_{t} N_{r}$ operations for the $F$ supersymbols 192 of the transmission frame. The selective CR of DLT-CI involves 193 the additional multiplication of the precoding matrix with $\mathbf{Q}_{\phi}^{k}$ at 194 every symbol period, with complexity of $N_{t} N_{r}^{2}$. At the receiver, all 195 techniques require a demodulation stage that involves $M$ comparisons 196 for $M$-order modulation for each of the $N_{r}$ RAs. The DLT and 197 DLT-CI require an additional stage for the detection of the spatial 198 symbol, which, from (10), involves $N_{a}$ complex multiplications and $199 N_{a}$ complex additions for each antenna combination out of the $\left(\begin{array}{l}N_{r} \\ N_{a}\end{array}\right)$ 200 combinations in total.

201 Fig. 1 shows the complexity of SMX, DLT, and the proposed 202 DLT-CI for a system with $N_{t}=8$ TAs and increasing numbers of 203 RAs $N_{r}$, with $N_{a}=N_{r} / 2$. For reference, we have assumed a Long204 Term Evolution (LTE) Type-2 time-division duplexing (TDD) frame 205 structure for which $F=70$, as detailed in [17]. A slow-fading channel 206 is assumed where the channel remains constant for the duration of 207 the frame. It can be seen that the proposed DLT-CI has increased complexity compared with DLT. However, it will be shown in the 208 following results that the improved performance for DLT-CI is worth 209 the added complexity.

\section{ERROR PROBABILITY}

The error probability of the proposed scheme can be described by 212 means of the pairwise error probability (PEP) $\mathcal{P}\left(\mathbf{s}_{m}^{k} \rightarrow \mathbf{s}_{n}^{l}\right)$. By the 213 use of the union bound, the average bit error probability $P_{e}$ can be 214 expressed as [13]

$$
P_{e} \leq \frac{1}{b} E\left\{\sum_{\mathbf{s}_{m}^{k} \in \mathcal{B}} \sum_{\mathbf{s}_{n}^{l} \in \mathcal{B} \neq \mathbf{s}_{m}^{k}} d\left(\mathbf{s}_{m}^{k}, \mathbf{s}_{n}^{l}\right) \mathcal{P}\left(\mathbf{s}_{m}^{k} \rightarrow \mathbf{s}_{n}^{l}\right)\right\}
$$

where $d\left(\mathbf{s}_{m}^{k}, \mathbf{s}_{n}^{l}\right)$ is the Hamming distance between the bit representa- 216 tions of the symbols $\mathbf{s}_{m}^{k}, \mathbf{s}_{n}^{l}$, and $\mathcal{B}$ is the supersymbol constellation 217 defined as the union of the spatial-domain constellation and of the 218 classic modulation constellation. The PEP can further be decomposed 219 into the PEP for the spatial symbol $\mathcal{P}\left(s_{m_{i}}^{k} \rightarrow s_{m_{i}}^{l}\right)$ and the PEP 220 $\mathcal{P}\left(s_{m_{i}}^{k} \rightarrow s_{n_{i}}^{k}\right)$ of the modulated symbol. These obey the following 221 lemmas.

Lemma 1: The PEP of the spatial symbol for the DLT-CI transmis- 223 sion obeys

$$
\mathcal{P}\left(s_{m_{i}}^{k} \rightarrow s_{m_{i}}^{l}\right)=Q\left(\frac{\beta}{\sqrt{2 \sigma^{2}}} \cdot \frac{\sqrt{P_{i}}-\sqrt{P_{L}}}{2}\right)
$$

where $Q($.$) denotes the Gaussian Q-function.$

Lemma 2: The PEP for the $M$-order phase-shift keying ( $M$-PSK) 226 modulated symbol, which is the focus of this work, follows:

$$
\mathcal{P}\left(s_{m_{i}}^{k} \rightarrow s_{n_{i}}^{k}\right)=Q\left(\beta \sqrt{\frac{P_{i}}{2 \sigma^{2}} \log _{2}(M) \sin \frac{\pi}{M}}\right) .
$$

Both the above expressions can be straightforwardly derived by adapt- 228 ing the methodology introduced in [17] for the proposed scenario. It is 229 the PEP in (14) that is enhanced for the proposed scheme by allowing 230 constructive interference to increase $P_{i}$. The tightness of the above- 231 described bound is validated in Section V.

\section{NUMERICAL RESULtS}

To evaluate the benefits of the proposed technique, this section 234 presents Monte Carlo simulations of the proposed DLT-CI in compar- 235 ison to conventional approaches. As the superiority of conventional 236 DLT over the most relevant SM and SMX approaches was thoroughly 237 validated in [17] and to limit the congestion in the following graphs, 238 here, we only use conventional DLT and SMX as a reference for 239 comparison. The channel impulse response is assumed to be perfectly 240 known at the transmitter for all techniques. Without loss of generality, 241 unless stated otherwise, we assume that the transmit power is restricted 242 to $P=1$. MIMO systems with up to eight TAs employing quaternary 243 phase-shift keying (QPSK), 8-PSK, and 16-PSK modulation are ex- 244 plored, albeit it is plausible that the benefits of the proposed technique 245 extend to larger-scale systems and higher-order modulation. For DLT 246 and DLT-CI, we focus on the case $N_{a}=N_{r} / 2$, which provides the 247 highest BE [17].

In Figs. 2 and 3, we show the BER with increasing signal-to- 249 noise ratio (SNR) for QPSK and 8-PSK, respectively. To complete 250 our comparisons, for both scenarios in the figure, we also show the 251 cases where the symbol modulation order used for SMX is increased 252 for some of the spatial streams to achieve the same BE values of 253 $\epsilon=10$ and $\epsilon=14$ with the proposed DLT, for QPSK and 8-PSK, 254 respectively. The figures also show the theoretical bound of (13) on 255 


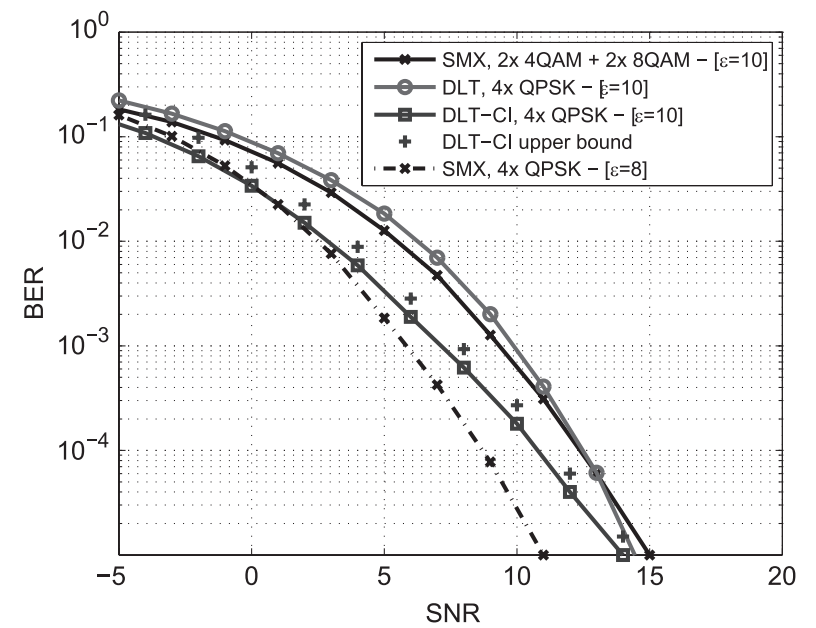

Fig. 2. BER versus SNR for a $(8 \times 4)$ MIMO with SMX, DLT, and DLT-CI; QPSK modulation.

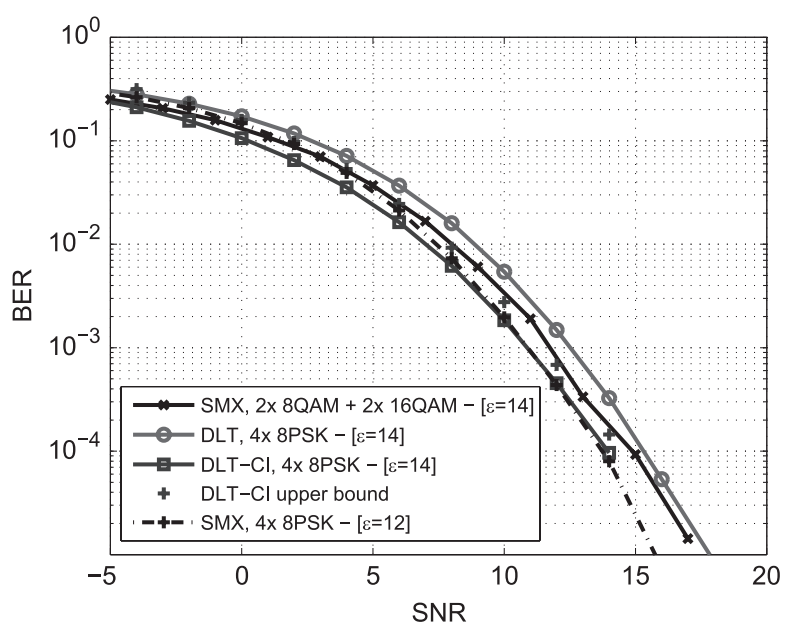

Fig. 3. BER versus SNR for a $(8 \times 4)$ MIMO with SMX, DLT, and DLT-CI; 8-PSK modulation.

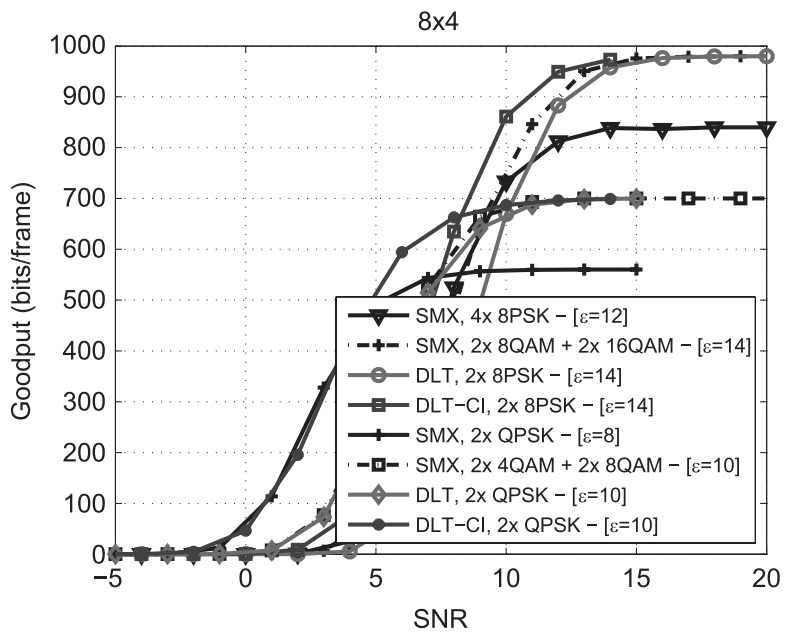

Fig. 4. Throughput versus SNR for a $(8 \times 4)$ MIMO with SMX, DLT, and DLT-CI.

256 the error probability, which closely matches our simulation results in 257 both cases. Clearly, the DLT scheme has an inferior BER performance 258 compared with SMX due to the additional spatial streams, which is the

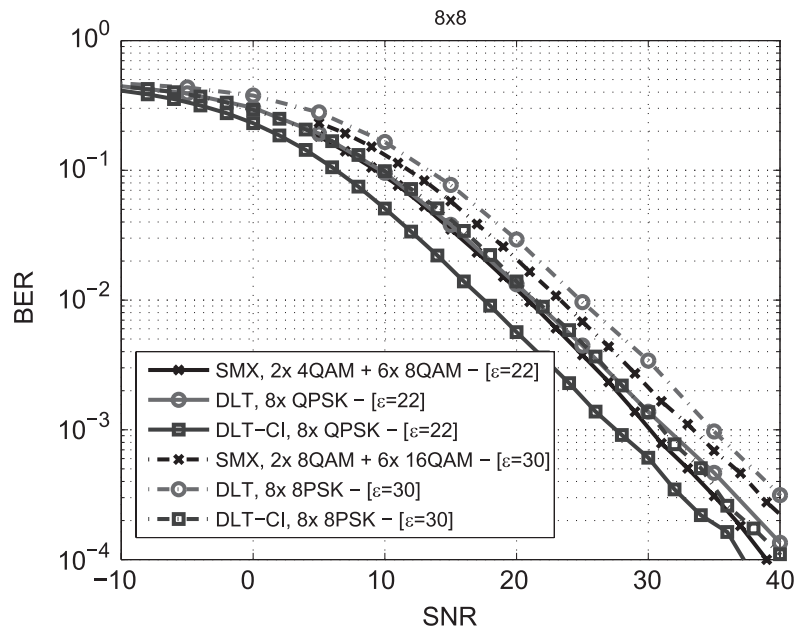

Fig. 5. BER versus SNR for a $(8 \times 8)$ MIMO with SMX, DLT, and DLT-CI.

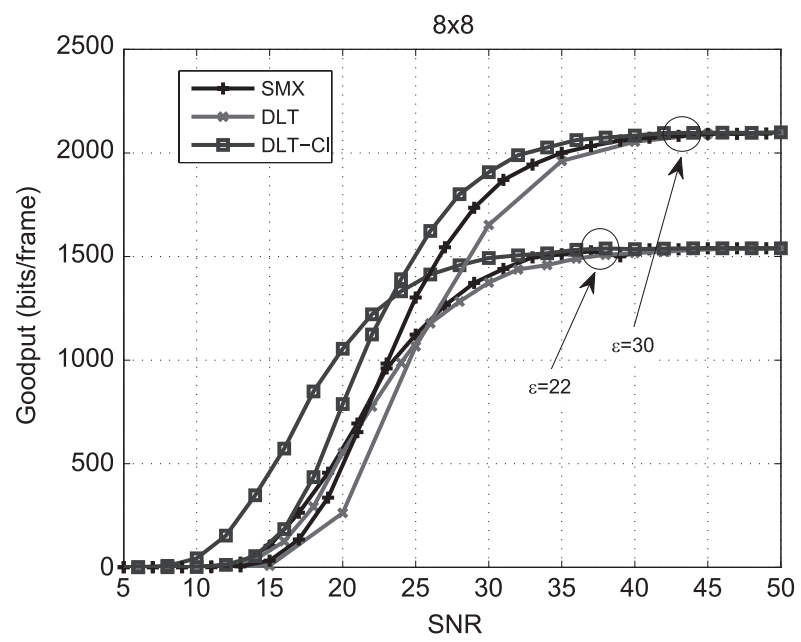

Fig. 6. Throughput versus SNR for a $(8 \times 8)$ MIMO with SMX, DLT, and DLT-CI.

price paid for its improved BE. DLT-CI outperforms both SMX and 259 DLT as an explicit benefit of the constructive interference exploited 260 as useful signal power, both in the modulated symbol detection and 261 in the formation of the different power levels employed for the spatial 262 symbol transmission. The improved BE of DLT-CI is demonstrated in 263 Fig. 4, where goodput versus SNR is depicted for the same $(8 \times 4) 264$ MIMO scenario. The goodput here is defined as $R=\epsilon F\left(1-P_{e}\right)^{F}, 265$ where $P_{e}$ is the bit error probability [17]. For reference, we have 266 assumed an LTE Type-2 TDD frame structure for which we have 267 $F=70$, as detailed in [17]. Clearly, DLT-CI provides the best goodput 268 performance among the schemes explored.

Our performance comparison is extended to the $(8 \times 8)$ MIMO 270 system in Figs. 5 and 6. The BER performance with increasing SNR is 271 shown in Fig. 5 for the $(8 \times 8)$ MIMO system where it can be seen that 272 DLT-CI outperforms both SMX and DLT. Fig. 6 shows the goodput 273 with increasing SNR, where, again, it can be observed that DLT-CI 274 provides the best goodput.

\section{CONCLUSION}

An enhanced dual-layered DL transmission scheme has been pro- 277 posed, which combines traditional MIMO SMX with RSM. The 278 proposed scheme improves upon conventional DLT by allowing con- 279 structive interference to carry spatial information, as opposed to the 280 
281 fixed power-level split of the conventional DLT in [17]. Our results show 282 that by allowing constructive interference to separate the power levels 283 and convey the spatial symbol, the proposed DLT-CI improves the BE 284 of SMX while, at the same time, the increased power levels of the sub285 set of symbols improve the average error performance of the system.

\section{REFERENCES}

1] C. B. Peel, B. M. Hochwald, and A. L. Swindlehurst, "A vectorperturbation technique for near-capacity multiantenna multiuser communication-Part I: Channel inversion and regularization," IEEE Trans. Commun., vol. 53, no. 1, pp. 195-202, Jan. 2005.

[2] C. Masouros, "Correlation rotation linear precoding for MIMO broadcast communications," IEEE Trans. Signal Process., vol. 59, no. 1, pp. 252-262, Jan. 2011.

[3] R. Mesleh, H. Haas, S. Sinanovic, C. W. Ahn, and S. Yun, "Spatial Modulation," IEEE Trans. Veh. Technol., vol. 57, no. 4, pp. 2228-2241, Jul. 2008.

[4] M. Di Renzo, H. Haas, A. Ghrayeb, S. Sugiura, and L. Hanzo, "Spatial modulation for generalized MIMO: Challenges, opportunities, and implementation," Proc. IEEE, vol. 102, no. 1, pp.56-103, Jan. 2014.

[5] Y. Ping, M. Di Renzo, X. Yue, L. Shaoqian, and L. Hanzo, "Design guidelines for spatial modulation," IEEE Commun. Surveys Tuts., vol. 17, no. 1, pp. 6-26, 1st Quart. 2015.

[6] M. Di Renzo and H. Haas, "On transmit diversity for spatial modulation MIMO: Impact of spatial constellation diagram and shaping filters at the transmitter," IEEE Trans. Veh. Technol., vol. 62, no. 6, pp. 2507-2531, Jul. 2013.

[7] P. Yang et al., "Star-QAM signaling constellations for spatial modulation," IEEE Trans. Veh. Technol., vol. 63, no. 8, pp. 3741-3749, Oct. 2014.

[8] S. Sugiura and L. Hanzo, "On the joint optimization of dispersion matrices and constellations for near-capacity irregular precoded space-time shift keying," IEEE Trans. Wireless Commun., vol. 12, no. 1, pp. 380-387, Jan. 2013.

[9] M. Maleki, H. Bahrami, S. Beygi, M. Kafashan, and N. H. Tran, "Space modulation with CSI: Constellation design and performance evaluation," IEEE Trans. Veh. Technol., vol. 62, no. 4, pp. 1623-1634, May 2013.

10] X. Guan, Y. Cai, and W. Yang, "On the mutual information and precoding for spatial modulation with finite alphabet," IEEE Wireless Commun. Lett., vol. 2, no. 4, pp. 383-386, Aug. 2013.
[11] C. Masouros, "Improving the diversity of spatial modulation in MISO 320 channels by phase alignment," IEEE Commun. Lett., vol. 18, no. 5, 321 pp. 729-732, May 2014.

[12] M. Di Renzo and H. Haas, "Improving the performance of Space Shift 323 Keying (SSK) modulation via opportunistic power allocation," IEEE 324 Commun. Lett., vol. 14, no. 6, pp. 500-502, Jun. 2010.

[13] C. Masouros and L. Hanzo, "Constellation-randomization achieves 326 transmit diversity for single-RF spatial modulation," IEEE Trans. Veh. 327 Technol., to be published.

[14] A. Garcia, C. Masouros, and L. Hanzo, "Pre-scaling optimization for 329 space shift keying based on semidefinite relaxation," IEEE Trans. Com- 330 mun., vol. 63, no. 11, pp. 4231-4243, Nov. 2015.

[15] R. Zhang, L. Yang, and L. Hanzo, "Generalised pre-coding aided spa- 332 tial modulation," IEEE Trans. Wireless Commun., vol. 12, no. 11, 333 pp. 5434-5443, Nov. 2013.

[16] L.-L. Yang, "Transmitter preprocessing aided spatial modulation 335 for multiple-input multiple-output systems," in Proc. IEEE $73 \mathrm{rd} 336$ VTC-Spring, May 15-18, 2011, pp. 1-5.

[17] C. Masouros and L. Hanzo, "Dual layered downlink MIMO transmis- 338 sion for increased bandwidth efficiency," IEEE Trans. Commun., to be 339 published.

[18] C. Masouros and E. Alsusa, "Soft linear precoding for the downlink of 341 DS/CDMA communication systems," IEEE Trans. Veh. Technol., vol. 59, 342 no. 1, pp. 203-215, Jan. 2010.

[19] C. Masouros and E. Alsusa, "Dynamic linear precoding for the exploita- 344 tion of known interference in MIMO broadcast systems," IEEE Trans. 345 Wireless Commun., vol. 8, no. 3, pp. 1396-1404, Mar. 2009.

346

[20] C. Masouros, T. Ratnarajah, M. Sellathurai, C. Papadias, and A. Shukla, 347 "Known interference in wireless communications: A limiting factor or a 348 potential source of green signal power?" IEEE Commun. Mag., vol. 51, 349 no. 10 , pp. $162-171$, Oct. 2013.

[21] G. Zheng et al., "Rethinking the role of interference in wireless networks," 351 IEEE Commun. Mag., vol. 52, no. 11, pp. 152-158, Nov. 2014.

[22] C. Masouros and E. Alsusa, "Two-stage transmitter precoding based on 353 data-driven code hopping and partial zero forcing beamforming for MC- 354 CDMA communications," IEEE Trans. Wireless Commun., vol. 8, no. 7, 355 pp. 3634-3645, Jul. 2009.

[23] C. Masouros and G. Zheng, "Exploiting known interference as green sig- 357 nal power for downlink beamforming optimization," IEEE Trans. Signal 358 Process., vol. 63, no. 14, pp. 3668-3680, Jul. 2015.

[24] C. Masouros, M. Sellathurai, and T. Ratnarajah, "Vector perturbation 360 based on symbol scaling for limited feedback MISO downlinks," IEEE 361 Trans. Signal. Process., vol. 62, no. 3, pp. 562-571, Feb. 1, 2014. 


\section{AUTHOR QUERIES}

\section{AUTHOR PLEASE ANSWER ALL QUERIES}

AQ1 = Please provide the complete current affiliation of author C. Masouros and check if the provided affiliation of author L. Hanzo is correct.

AQ2 = Uncited Figure 4. Figure 6 was changed to Figure 4. Please check if correct.

AQ3 = Please provide publication update in Ref. [13].

AQ4 = Please provide publication update in Ref. [17].

\section{END OF ALL QUERIES}

